\title{
Relation between the generalized acoustic analogy and Lilley's contributions to aeroacoustics
}

\author{
M.E. Goldstein ${ }^{*}$ \\ NASA Glenn Research Center 21000 Brookpark Rd, Cleveland OH, 44135
}

Submitted March 17, 2009; Revised September 21, 2009; Accepted January 12, 2010

\begin{abstract}
This paper reviews Lilley's reformulation of Lighthill's equation and shows that it can be obtained as a special case of a much more general acoustic analogy. It also shows how this generalized analogy can be used to eliminate some of the difficulties that arise when more conventional parallel flow analogies are applied to high speed jets. And, finally, some recent applications of these ideas are discussed.
\end{abstract}

\section{INTRODUCTION}

It's with considerable pleasure that I write this paper in honor of Geoffrey Lilley who is so unassuming that it is easy to forget how important his contributions to Aeroacoustics actually are. After thinking about this for awhile, it occurred to me that the best way to acknowledge the importance of those contributions would be amplify some of his results by embedding them in a more general context. So I started with his clarification of the so called "entropy" source term $\delta_{i j}\left(p-c_{0}^{2} \rho\right)$ in the Lighthill stress tensor

$$
\tau_{i j} \equiv-\rho v_{i} v_{j}+\delta_{i j}\left(p-c_{o}^{2} \rho\right)+\sigma_{i j}
$$

that appears on the right hand side of Lighthill's $(1952,1954)$ equation

$$
\frac{\partial^{2} \rho^{\prime}}{\partial t^{2}}-c_{o}^{2} \frac{\partial^{2} \rho^{\prime}}{\partial x_{j} \partial x_{j}}=-\frac{\partial^{2}}{\partial x_{i} \partial x_{j}} \tau_{i j}
$$

where $t$ denotes the time, $\boldsymbol{x}=\left\{x_{i}\right\}$ denote Cartesian coordinates, $\rho$ denotes the density, $v_{i}$ denotes the fluid velocity, $p$ denotes the pressure, $\sigma_{i j}$ denotes the viscous stress, the subscript zero refers to the uniform background state at infinity, with $c_{0}$ being its sound

*Email address: marvin.e.goldstein@nasa.gov 
speed and $\rho^{\prime} \equiv \rho-\rho_{0}$ being the density fluctuation. Lilley $(1996,2003)$ introduced a modification of Lighthill's equation that separates this term into isentropic and nonisentropic components, which is important because the non-isentropic component is proportional to the heat flux vector $q_{i}$ and the viscous work term $\sigma_{i j} v_{j}$ that are usually neglected at the high Reynolds numbers where jet noise becomes important.

While there are other ways of accomplishing this that lead to simpler results (Goldstein, 2001) an important advantage of Lilley's approach is that it can be extended in a natural way to a more general formulation that promises to become increasingly important as computational capability continues to increase (Bodony and Lele, 2002, 2003, 2005, Karabasov et al, 2008). The present paper shows how Lilley's revision of Lighthill's equation can be obtained as a special case of this more general result. Some recent applications of this generalized approach are also discussed.

\section{LILLEY'S MODIFICATION OF LIGHTHILL'S EOUATION}

Lilley began by rewriting Lighthill's equation in terms of the pressure to obtain

$$
\frac{1}{c_{0}^{2}} \frac{\partial^{2} p}{\partial t^{2}}-\frac{\partial^{2} p}{\partial x_{j} \partial x_{j}}=\frac{\partial^{2}}{\partial x_{i} \partial x_{j}}\left(\rho v_{i} v_{j}-\sigma_{i j}\right)+\frac{\partial^{2}}{\partial t^{2}}\left(\frac{p-c_{0}^{2} \rho}{c_{0}^{2}}\right)
$$

He then noted that the energy equation can be written in terms of the stagnation enthalpy

$$
h_{s} \equiv h+\frac{1}{2} v^{2}
$$

as

$$
\frac{\partial\left(\rho h_{s}-p\right)}{\partial t}+\frac{\partial}{\partial x_{i}} \rho v_{i} h_{s}=-\frac{\partial}{\partial x_{i}}\left(q_{i}-\sigma_{i j} v_{j}\right)
$$

where $h$ denotes the enthalpy. The continuity equation

$$
\frac{\partial \rho}{\partial t}+\frac{\partial}{\partial x_{j}} \rho v_{j}
$$

can be used to write this as

$$
\frac{\partial\left[\rho\left(h_{s}-h_{0}\right)-p\right]}{\partial t}+\frac{\partial}{\partial x_{i}} \rho v_{i}\left(h_{s}-h_{0}\right)=-\frac{\partial}{\partial x_{i}}\left(q_{i}-\sigma_{i j} v_{j}\right)
$$


where $h_{0}$ has the obvious meaning. But since $h_{0}$ is equal to $c_{0}^{2} /(\gamma-1)$, where $\gamma$ is the specific heat ratio, and $\rho h-p$ is equal to $h_{0} p / c_{0}^{2}$ for an ideal (and perfect) gas this equation can be written as

$$
\frac{\partial}{\partial t}\left[\frac{p-c_{0}^{2} \rho+(\gamma-1) \rho v^{2} / 2}{c_{0}^{2}}\right]+\frac{\partial}{\partial x_{i}} \rho v_{i}\left(\frac{h_{s}-h_{0}}{h_{0}}\right)=-\frac{1}{h_{0}} \frac{\partial}{\partial x_{i}}\left(q_{i}-\sigma_{i j} v_{j}\right)
$$

in that case. A flow will be isentropic, i.e., the convective derivative of its entropy will vanish (Yih, 1977, p. 255), when the viscous and heat conduction effects (i.e., the diffusive effects) are zero. So the right hand side of [2.6] will be equal to zero and the left hand side will vanish in that case. Equation [2.6], therefore, implies that $\partial\left[\left(p-c_{0}^{2} \rho\right) / c_{0}^{2}\right] / \partial t$ can be divided into isentropic and non-isentropic (or in Lilley's words diffusive and non-diffusive) components. And inserting it into [2.1] shows that (Lilley, 1996, 2003)

$$
\begin{aligned}
& \frac{1}{c_{0}^{2}} \frac{\partial^{2} p}{\partial t^{2}}-\frac{\partial^{2} p}{\partial x_{j} \partial x_{j}}=\frac{\partial^{2}}{\partial x_{i} \partial x_{j}}\left(\rho v_{i} v_{j}-\sigma_{i j}\right) \\
& -\frac{\partial^{2}}{\partial t \partial x_{i}}\left[\frac{\rho v_{i}\left(h_{s}-h_{0}\right)+q_{i}-\sigma_{i j} v_{j}}{h_{0}}\right]-\frac{\partial^{2}}{\partial t^{2}}\left[\frac{(\gamma-1) \rho v^{2}}{2 c_{0}^{2}}\right]
\end{aligned}
$$

The advantage of this equation over the original Lighthill result is that the diffusive ( $2^{\text {nd }}$ term on right hand side) and non-diffusive ( $3^{\text {rd }}$ term on right hand side) components of the source term are now explicitly displayed. The main disadvantage is that Lighthill's simple quadrupole source is now replaced by a combination of quadrupole, dipole and monopole sources. Lighthill (1954) emphasized the importance of displaying the correct multipole order of the source term. He argued that the inclusion of extraneous lower order multipole components that are more efficient than the higher order multipole components at low frequencies could lead to an over estimation of the radiated sound in the low frequency limit. Inclusion of the dipole term appears to be unavoidable, but the monopole term can be eliminated by introducing the new dependent variable

$$
p_{e} \equiv p+\frac{\gamma-1}{2} \rho v^{2}
$$

to obtain the somewhat simpler result

$$
\frac{1}{c_{0}^{2}} \frac{\partial^{2} p_{e}}{\partial t^{2}}-\frac{\partial^{2} p_{e}}{\partial x_{j} \partial x_{j}}=-\frac{\partial^{2} e_{i j}}{\partial x_{i} \partial x_{j}}+\frac{1}{c_{0}^{2}} \frac{\partial^{2} e_{4 j}}{\partial t \partial x_{j}}
$$


where the source strength $e_{\lambda i}$ is now given by the $4 \times 3$ dimensional "Lilley's Stress Tensor"

$$
e_{\lambda i} \equiv-\rho v_{\lambda} v_{i}+\frac{\gamma-1}{2} \delta_{\lambda i} \rho v^{2}+\sigma_{\lambda i} \quad \text { for } \lambda=1,2,3,4
$$

with

$$
v_{4} \equiv(\gamma-1)\left(h_{s}-h_{0}\right), \quad \text { and } \quad \sigma_{4 i} \equiv-(\gamma-1)\left(q_{i}-\sigma_{i j} v_{j}\right)
$$

(The Greek indices $\lambda$ and $\kappa$ will always range from 1 to 4 , all other Greek indices will range from 1 to 5 and Latin indices will range from 1 to 3.).

Lilley dealt with the monopole term by arguing that the relatively small $(\gamma-1) / 2$ multiplicative factor should cause it to be negligibly small. But Goldstein and Leib (2008) found that it actually makes a significant contribution to the $90^{\circ}$ sound field when the turbulence is assumed to be quasi-normal and axysymmetric and this probably remains true even when it is not quasi-normal. It is now generally believed that the remaining enthalpy term $\left(\partial^{2} e_{4 j} / \partial t \partial x_{j}\right) / c_{0}^{2}$ is an important sound source in heated jets and significant effort is underway to develop appropriate models for this quantity (Khavaran and Kenzakowski, 2007).

Equation [2.9] can be made to resemble Lighthill's equation even more closely by introducing the new dependent variable

$$
x_{4} \equiv-c_{0}^{2} t
$$

to obtain

$$
\frac{1}{c_{0}^{2}} \frac{\partial^{2} p_{e}}{\partial t^{2}}-\frac{\partial^{2} p_{e}}{\partial x_{j} \partial x_{j}}=-\frac{\partial^{2} e_{\lambda j}}{\partial x_{\lambda} \partial x_{j}}
$$

where the summation convention is now being used for the Greek as well as the Latin indices. The non-linear dependent variable $p_{e}$ (or equivalently $p_{e}-p_{0}$ ) causes no particular difficulty here because it reduces to the ordinary pressure fluctuation $p-p_{0}$ in the far field where the sound is to be calculated.

\section{A GENERALIZED APPROACH}

Lilley was also one of the first to emphasize the importance of removing all non-sound generating terms from the source function in order to obtain accurate predictions of the sound field. This is very difficult to do in practice, but can be approached more and more closely with increased computational effort. The appropriate acoustic analogy equations can be obtained by moving more of these silent terms to the left hand side of the NavierStokes equations and expressing the result as a set of formally linear equations with nonlinear dependent variables and source terms. 
This can be accomplished by dividing the dependent variables in the Navier Stokes equations, i.e. the density, pressure, velocity and enthalpy into, say, a "base flow" component, which is denoted by the over-bars and tildes and "residual" components, which are essentially defined by the equations

$$
\rho=\bar{\rho}+\rho^{\prime}, \quad p=\bar{p}+p^{\prime}, \quad h=\tilde{h}+h^{\prime} \quad v_{i}=\tilde{v}_{i}+v_{i}^{\prime},
$$

and requiring that the base flow components satisfy the usual hyperbolic conservation laws, which can be written fairly compactly as (Goldstein and Leib, 2008)

$$
\begin{gathered}
D_{o} \bar{\rho}=0 \\
D_{o} \bar{\rho} \tilde{v}_{i}+\frac{\partial \bar{p}_{e}}{\partial x_{i}}=\frac{\partial}{\partial x_{j}} \tilde{e}_{i j} \\
D_{o}\left(\frac{\gamma \bar{p}_{e}}{\gamma-1}+\frac{\bar{\rho}}{2} \tilde{v}^{2}\right)-\frac{\partial \bar{p}_{e}}{\partial t}=\frac{\partial}{\partial x_{i}}\left(\frac{\tilde{e}_{4 i}}{\gamma-1}+\tilde{e}_{i j} \tilde{v}_{j}\right)
\end{gathered}
$$

where the base flow variables, as well as the original Navier Stokes variables are assumed to satisfy an ideal gas law equation of state, $\bar{p}_{e}$ denotes a pressure-like variable that can differ from the thermodynamic pressure $\bar{p}, \tilde{e}_{\lambda j}$ denotes an arbitrary $4 \times 3$ dimensional stress tensor and

$$
D_{o} f \equiv \frac{\partial f}{\partial t}+\frac{\partial}{\partial x_{j}}\left(\tilde{v}_{j} f\right)
$$

for any function $f$. These equations include, among other things, the Euler equations, the Navier Stokes equations themselves, and even the Favre filtered Navier Stokes equations with very general space-time filtering (Aldama, 1990, Goldstein, 2005, 2008).

It can now be shown (Goldstein, 2000, 2002, 2003, see also Goldstein and Leib, 2008) that the remaining residual variables are determined by the five formally linear equations

$$
\begin{gathered}
D_{o} \rho^{\prime}+\frac{\partial}{\partial x_{j}} u_{j}=0 \\
D_{o} u_{i}+u_{j} \frac{\partial \tilde{v}_{i}}{\partial x_{j}}+\left(\frac{\partial}{\partial x_{i}} p_{e}^{\prime}+\rho^{\prime} X_{i}\right)=\frac{\partial}{\partial x_{j}} e_{i j}^{\prime \prime}
\end{gathered}
$$

and

$$
D_{o} p_{e}^{\prime}+\frac{\partial}{\partial x_{j}} \widetilde{c^{2}} u_{j}+(\gamma-1)\left(p_{e}^{\prime} \frac{\partial \tilde{v}_{j}}{\partial x_{j}}+u_{i} X_{i}\right)=\frac{\partial}{\partial x_{j}} e_{4 j}^{\prime \prime}+(\gamma-1) e_{i j}^{\prime \prime} \frac{\partial \tilde{v}_{i}}{\partial x_{j}}
$$


where

$$
\widetilde{c^{2}} \equiv \gamma \bar{p} / \bar{\rho}
$$

is the square of the base flow sound speed and

$$
X_{i} \equiv\left(\frac{\partial}{\partial \tau}+\tilde{v}_{j} \frac{\partial}{\partial y_{j}}\right) \tilde{v}_{i}=\frac{1}{\bar{\rho}} \frac{\partial}{\partial y_{i}}\left(\tilde{e}_{i j}-\delta_{i j} \bar{p}_{e}\right)
$$

is the base flow advection vector.

The Navier-Stokes equations have been put into this linearized hyperbolic conservation law form by introducing the non-linear dependent variables

$$
\begin{aligned}
p_{e}^{\prime} \equiv p-\bar{p}_{e}+\frac{\gamma-1}{2} \rho v^{\prime 2} & =\left(p^{\prime}+\frac{\gamma-1}{2} \rho v^{\prime 2}\right)+\bar{p}-\bar{p}_{e} \\
\mathrm{u}_{\mathrm{i}} & \equiv \rho \mathrm{v}_{\mathrm{i}}^{\prime},
\end{aligned}
$$

as well as in the quadratically non-linear source strengths

$$
e_{\lambda i}^{\prime \prime} \equiv e_{\lambda i}^{\prime}-\tilde{e}_{\lambda i} \quad \lambda=1,2,3,4
$$

where $e_{\lambda i}^{\prime}$ denotes the generalized "Lilley Stress Tensor"

$$
e_{\lambda i}^{\prime} \equiv-\rho v_{\lambda}^{\prime} v_{i}^{\prime}+\frac{\gamma-1}{2} \delta_{\lambda i} \rho v^{\prime 2}+\sigma_{\lambda i}
$$

with

$$
v_{4}^{\prime} \equiv(\gamma-1) h_{s}^{\prime}, \quad \sigma_{4 i} \equiv-(\gamma-1)\left(q_{i}-\sigma_{i j} v_{j}^{\prime}\right)
$$

and

$$
h_{s}^{\prime} \equiv h^{\prime}+\frac{1}{2} v^{\prime 2}
$$

is the residual stagnation enthalpy (i.e. the difference between the stagnation enthalpy and the base flow stagnation enthalpy) in a reference frame moving with the local base flow velocity $\widetilde{v}_{i}$. 
The nonlinear variable [3.12] is crucial but, as in the Lilley formalism, the nonlinear variable [3.11] is mainly introduced to simplify the result. Since the velocity variable is already non-linear there is no reason to preclude this potentially very significant (see Goldstein and Leib, 2008) reduction in complexity by artificially requiring that the pressure variable be linear. The moving frame residual stagnation enthalpy $h_{s}^{\prime}$ was introduced because order of magnitude estimates (Goldstein and Leib, 2008) suggest that $v_{4}^{\prime} \equiv(\gamma-1) h_{s}^{\prime} \quad$ will be negligible compared to $v_{i}^{\prime}$ in cold (i.e. unheated) jets at $O$ (1) Mach numbers-which means that $e^{\prime}{ }_{4 i}$ will then be negligible compared to $e_{j i}^{\prime}$ and that the source term can, therefore, be considerably simplified. But recent RANS computotions (Khavaran and Kenzakowski, 2007) suggest that it may be the fixed frame residual stagnation enthalpy $h_{s}^{\prime}+\tilde{v}_{i} v_{i}^{\prime}$ that is negligibly small compared to $v_{i}^{\prime}$ in cold (i.e. unheated) jets at very high Mach numbers. It would then be better to define $v_{4}^{\prime}$ by $v_{4}^{\prime} \equiv(\gamma-1)\left(h_{s}^{\prime}+\tilde{v}_{i} v_{i}^{\prime}\right)$ and, as a consequence, change the right side of [3.8] to $\partial e_{4 j}^{\prime \prime} / \partial x_{j}-(\gamma-1) \tilde{v}_{i} \partial e_{i j}^{\prime \prime} / \partial x_{j}$.

But at present the only safe course of action would be to retain the $4^{\text {th }}$ component $e_{4 i}^{\prime \prime}$ even for cold jets-in which case the precise definition of $v_{4}^{\prime}$ would be largely irrelevant.

Equations [3.6] to [3.8] can be written more compactly as

$$
L_{u v} u_{v}=D_{\lambda j}^{\mu} e_{\lambda j}^{\prime \prime} \quad \text { for } \quad \mu, v=1,2, \ldots 5
$$

where $L_{\mu v}$ and $D_{\lambda j}^{\mu}$ are simple first order linear operators (given explicitly in Goldstein, 2006) and $u_{v}$ denotes the five dimensional solution vector

$$
\left\{u_{v}\right\} \equiv\left\{u_{i}, p_{e}^{\prime}, \rho^{\prime}\right\}
$$

Interpretation of the right side of these equations as acoustic sources implies that that the left sides describe the interaction of this sound with as well as its propagation through the base flow. The formal linearity of the equations can now be exploited to separate out these base flow interaction effects (including the propagation of the sound through that flow) from the unsteady source fluctuations that produce the sound by using the $4^{\text {th }}$ component of the adjoint vector Green's function $g_{v \mu}^{a}(\boldsymbol{y}, \tau \mid \boldsymbol{x}, t)$, which is related to the $4^{\text {th }}$ component of the ordinary vector Green's function $g_{4 v}(\boldsymbol{x}, t \mid \boldsymbol{y}, \tau)$ by the reciprocity relation $g_{\mathrm{v} 4}^{a}(\boldsymbol{y}, \tau \mid \boldsymbol{x}, t)=g_{4 v}(\boldsymbol{x}, t \mid \boldsymbol{y}, \tau)$ and satisfies the adjoint equation

$$
\left(L_{\mu v}^{a}\right)_{\mathrm{y}, \tau} g_{v 4}^{a}(\boldsymbol{y}, \tau \mid \boldsymbol{x}, t)=\delta_{\mu 4} \delta(\boldsymbol{x}-\boldsymbol{y}) \delta(t-\tau)
$$

(Morse \& Fesbach, 1953, p. 870, Tam \& Auriault, 1998) where $L_{\mu \nu}^{a}$ is the adjoint of $L_{\mu \nu}$ to obtain the following formula for the pressure like variable $p_{e}^{\prime}$

$$
p_{e}^{\prime}(\boldsymbol{x}, t)=-\int_{V}^{\infty} \int_{-\infty}^{\infty} \gamma_{\lambda_{j}}(\boldsymbol{y}, \tau \mid \boldsymbol{x}, t) e_{\lambda_{j}}^{\prime \prime}(\boldsymbol{y}, \tau) d \mathbf{y} d \tau
$$


where the propagator $\gamma_{\mu j}(\boldsymbol{y}, \tau \mid \boldsymbol{x}, t)$ is related to $g_{\mu 4}^{a}(\boldsymbol{y}, \tau \mid \boldsymbol{x}, t)$ by

$$
\gamma_{\mu j}(\boldsymbol{x}, t \mid \boldsymbol{y}, \tau) \equiv \frac{\partial g_{\mu 4}^{a}(\boldsymbol{y}, \tau \mid \boldsymbol{x}, t)}{\partial y_{j}}-(\gamma-1) \delta_{\mu k} \frac{\partial \tilde{v}_{k}}{\partial y_{j}} g_{44}^{a}(\boldsymbol{y}, \tau \mid \boldsymbol{x}, t),
$$

$p_{e}^{\prime}(\boldsymbol{x}, t)$ reduces to the ordinary pressure fluctuation $p^{\prime}(\boldsymbol{x}, t)$ when $\boldsymbol{x}$ is in the far field and I am assuming, for simplicity, that all solid surface effect can be neglected. (But the result remains valid in the presence of fixed solid surfaces when viscous effects are neglected.)

Equation [3.17] is similar to [2.9] in that the entire source strength is described by a $4 \times 3$ dimensional tensor. In fact equations [3.2] to [3.4] reduce to the Euler equations (with $\bar{p}_{e}=\bar{p}$ ) when the base flow source strengths $\tilde{e}_{\lambda j}$ are set equal to zero. And these equations posses a family of solutions, which quite conveniently provides a good approximation to the actual mean flow field in boundary layers, turbulent air jets and other nearly parallel flows: namely the unidirectional transversely sheared mean flows

$$
\tilde{v}_{i}=\delta_{i 1} U\left(x_{2}, x_{3}\right), \bar{p}_{e}=\text { constant }, \bar{\rho}=\bar{\rho}\left(x_{2}, x_{3}\right) .
$$

where $U$ and $\bar{\rho}$ can be any functions of the indicated arguments. Equation [3.6], (i.e. the continuity equation) then decouples from the remaining four equations [3.7] and [3.8], which now become the inhomogeneous compressible Rayleigh equations (Betchov and Criminale 1967)

$$
\begin{gathered}
\frac{D_{o} u_{i}}{D t}+\delta_{i 1} u_{j} \frac{\partial U}{\partial x_{j}}+\frac{\partial p_{e}^{\prime}}{\partial x_{i}}=\frac{\partial}{\partial x_{j}} e_{i j}^{\prime} \\
\frac{D_{o} p_{e}^{\prime}}{D t}+\frac{\partial \widetilde{c^{2}} u_{j}}{\partial x_{j}}=\frac{\partial e_{4 j}^{\prime}}{\partial x_{j}}+(\gamma-1) e_{1 j}^{\prime} \frac{\partial U}{\partial x_{j}}
\end{gathered}
$$

where

$$
\frac{D_{o}}{D t} \equiv \frac{\partial}{\partial t}+U \frac{\partial}{\partial x_{1}}
$$

denotes the convective derivative.

It is now well known (Goldstein, 1976) that the velocity variable $u_{i}$ can be eliminated between these equations to obtain the inhomogeneous equation

$$
L p_{e}^{\prime}=\frac{D_{o}}{D t}\left(\frac{\partial}{\partial x_{i}} \widetilde{c^{2}} \frac{\partial e_{i j}^{\prime}}{\partial x_{j}}\right)-\frac{\partial U}{\partial x_{i}} 2 \widetilde{c^{2}} \frac{\partial^{2} e_{i j}^{\prime}}{\partial x_{1} \partial x_{j}}-\frac{D_{0}^{2}}{D t^{2}}\left[\frac{\partial e_{4 j}^{\prime}}{\partial x_{j}}+(\gamma-1) e_{1 j}^{\prime} \frac{\partial U}{\partial x_{j}}\right]
$$


where

$$
L \equiv \frac{D_{o}}{D t}\left(\frac{\partial}{\partial x_{i}} \widetilde{c^{2}} \frac{\partial}{\partial x_{i}}-\frac{D_{o}^{2}}{D t^{2}}\right)-2 \frac{\partial U}{\partial x_{j}} \frac{\partial}{\partial x_{1}} \widetilde{c^{2}} \frac{\partial}{\partial x_{j}}
$$

is the variable-density Pridmore-Brown (1958) or Rayleigh (Betchov and Criminale, 1967) operator. This is a form of Lilley's famous (1974) equation whose source term, as emphasized by Morris (personal communication), turns out to be somewhat more complicated than the original Rayleigh equation sources. It reduces exactly to equation [2.9] in the Lighthill limit

$$
U \rightarrow 0, \widetilde{c^{2}} \rightarrow c_{0}^{2}
$$

(after performing a trivial integration with respect to time). The analogue of [2.13] would be

$$
L p_{e}^{\prime}=\frac{D_{o}}{D t}\left(D_{\lambda} \widetilde{c^{2}} \frac{\partial e_{\lambda j}^{\prime}}{\partial x_{j}}\right)-\frac{\partial U}{\partial x_{i}} \widetilde{2 c^{2}}\left(\frac{\partial^{2} e_{i j}^{\prime}}{\partial x_{1} \partial x_{j}}+\frac{\gamma-1}{2} D_{4}^{2} \widetilde{c^{2}} e_{i 1}^{\prime}\right)
$$

where

$$
D_{\lambda} \equiv\left\{\begin{array}{c}
\frac{\partial}{\partial x_{i}}, \quad i=\lambda=1,2,3 \\
\frac{1}{\widetilde{c^{2}}} \frac{D_{o}}{D t}, \quad \lambda=4
\end{array}\right.
$$

While it can be argued that Lilley's equation (along with its variants) has served as the basis of most of the theoretical jet noise research over the past couple of decades, this result does have a number of issues. First of all the mean flow interaction effects have not been completely eliminated from the source function because $v_{i}^{\prime}$ still has a mean flow component. And secondly the source function contains a steady term that does not generate any sound and therefore does not belong in the source term. Another more serious difficulty is that the Rayleigh operator can support linear instability waves that grow without bound in this parallel mean flow model. But these homogeneous solutions contribute to the adjoint Greens' function and therefore to the propagator $\gamma_{\mu j}(\boldsymbol{y}, \tau \mid \boldsymbol{x}, t)$ when causality is imposed (Briggs, 1964, Bers, 1975, Goldstein and Leib, 2005) — causing the solution to become infinite far downstream in the flow.

Arguments against imposing causality (Mani 1976; Dowling, Ffowcs Williams \& Goldstein 1978) usually amount to asserting that it is unnecessary because it is not 


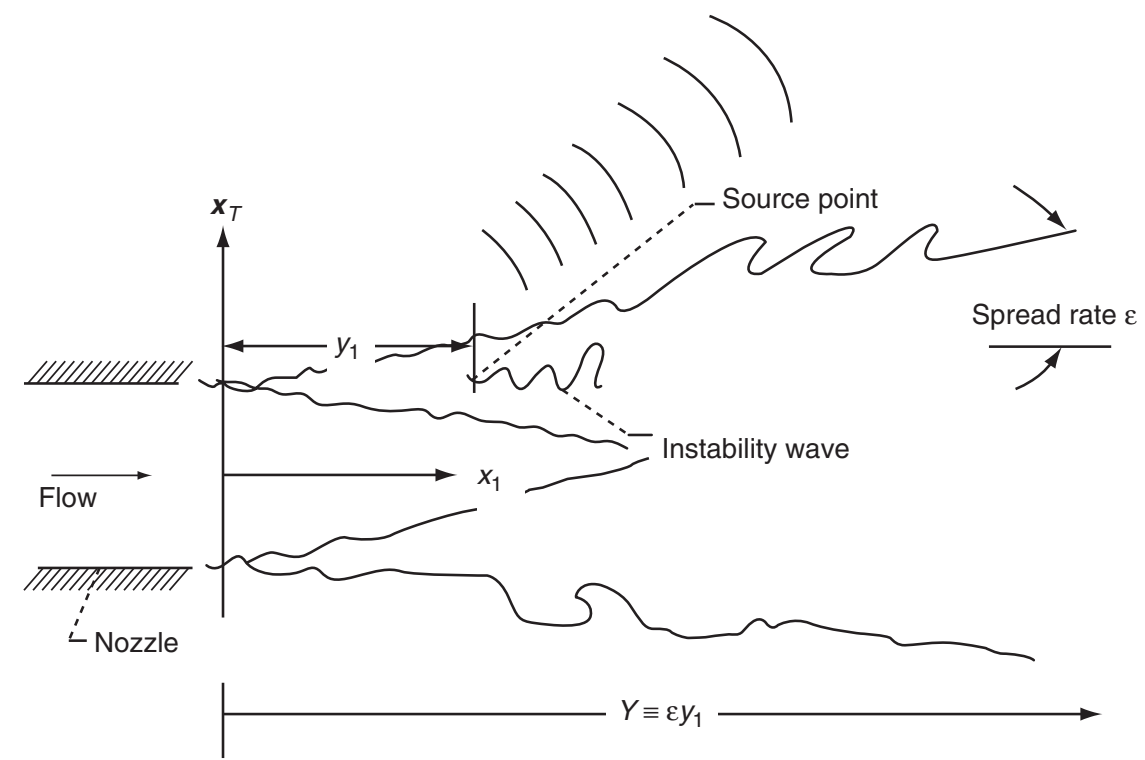

Figure 1: Jet flow configuration.

possible to identify an initial time before which the fluctuations (about the base flow) have been 'switched on' in an acoustic analogy approach and that a boundedness requirement can, therefore, be imposed on the solution. But the real problem that needs to be solved is not that of a doubly infinite parallel jet but that of a jet issuing from a nozzle as shown in figure 1 . The steady state (i.e., time stationary) solution to this problem can be made unique without considering initial conditions (i.e., causality) by imposing a Kutta condition at the nozzle lip.

Crighton and Leppington (1974) constructed the two dimensional Green's function for the analogous problem of a vortex sheet behind a semi-infinite plate, separating an ambient fluid from a stream moving with uniform speed (see also Crighton,1985, p. 436). They found that the imposition of the Kutta condition implies that causality will be satisfied in this case.

The doubly infinite parallel flow model amounts to neglecting the reflection and diffraction contributions to the Green's function in the true jet noise problem so that the remaining portion of the solution is likely to be the causal Greens function for the doubly infinite parallel flow. This suggest that the parallel flow Green's function should be required to satisfy causality in the jet noise problem-which means that it will not remain bounded.

Another serious difficulty at supersonic speeds is that the adjoint Greens function $g_{v \mu}^{a}$ has a singularity at the so called critical level which produces an even stronger nonintegrable singularity in the propagator $\gamma_{\mu j}(\boldsymbol{y}, \tau \mid \boldsymbol{x}, t)$ when the observation point $\boldsymbol{x}$ is 
in the far field-leading to the absurd conclusion that the radiated sound is infinitely loud.

\section{THE MEAN FLOW ANALOGY}

None of the difficulties would occur when if the base flow were taken to be the mean or time average of the actual flow-even when that flow is a nearly parallel shear flow. Because, for example, its slow divergence would cause the instability wave to eventually decay instead of growing without bound. And, more importantly at supersonic speeds, the flow would no longer posses a critical layer and would, therefore, not have a critical layer singularity.

This base flow is obtained be interpreting the over-bars in equations [3.2] to [3.4] as the time averages

$$
\bar{\bullet} \equiv \lim _{T \rightarrow \infty} \frac{1}{2 T} \int_{-T}^{T} \bullet(x, t) d t,
$$

and the tildes as the Favre averages

$$
\stackrel{\bullet}{\bullet}(\overline{\rho \bullet}) / \bar{\rho}
$$

in which case

$$
p_{e}^{\prime} \equiv p^{\prime}+\frac{\gamma-1}{2}\left(\rho v^{\prime 2}-\overline{\rho v^{\prime 2}}\right)
$$

and more importantly

$$
\tilde{e}_{v j}=\overline{e_{v j}^{\prime}}
$$

So the complete residual equation source strength is simply

$$
e_{v j}^{\prime \prime}=e_{v j}^{\prime}-\overline{e_{v j}^{\prime}}
$$

which means it has zero time average and therefore contains no steady component. The base flow equations are of course the usual Reynolds averaged Navier-Stokes equations. This form of the analogy completely removes the mean flow interaction effects from the source term, which is expected to significantly improve the medium and lower frequency predictions, but no steady base flow analogy can provide the requisite structure for inputting the long range turbulent scattering effects, which tend to become more important at the higher frequencies (and could eventually overwhelm the mean flow interaction effects at sufficiently high frequencies). 
But a major advantage of this analogy is that the Green's function solution [3.20] can be used to express the far field pressure autocovariance

$$
\overline{p^{2}}\left(\boldsymbol{x}, t_{0}\right) \equiv \frac{1}{2 T} \int_{-T}^{T} p^{\prime}(\boldsymbol{x}, t) p^{\prime}\left(\boldsymbol{x}, t+t_{0}\right) d t,
$$

as the convolution product

$$
\overline{p^{2}}(\boldsymbol{x}, t)=\int_{-\infty}^{\infty} \iint_{V} \bar{\gamma}_{\kappa j \lambda l}(\boldsymbol{x} \mid \boldsymbol{y} ; \boldsymbol{\eta}, t+\tau) R_{\kappa j \lambda l}(\boldsymbol{y} ; \boldsymbol{\eta}, \tau) d \boldsymbol{y} d \boldsymbol{\eta} d \tau
$$

of the " convolved propagator"

$$
\bar{\gamma}_{\kappa j \lambda l}(\boldsymbol{x} \mid \boldsymbol{y} ; \boldsymbol{\eta}, t+\tau) \equiv \int_{-\infty}^{\infty} \gamma_{\kappa j}\left(\boldsymbol{x} \mid \boldsymbol{y}, t_{1}+t+\tau\right) \gamma_{\lambda l}\left(\boldsymbol{x} \mid \boldsymbol{y}+\boldsymbol{\eta}, t_{1}\right) d t_{1},
$$

with the two point time-delayed correlation of the source strength

$$
R_{\kappa j \lambda l}(\boldsymbol{y} ; \boldsymbol{\eta}, \tau) \equiv \frac{1}{2 T} \int_{-T}^{T} e_{\kappa j}^{\prime \prime}\left(\boldsymbol{y}, \tau_{0}\right) e_{\lambda l}^{\prime \prime}\left(\boldsymbol{y}+\boldsymbol{\eta}, \tau_{0}+\tau\right) d \tau_{0}
$$

which is, in turn, related to the generalized Reynolds stress autocovariance tensor

$$
\boldsymbol{R}_{\lambda j \kappa l}(\boldsymbol{y}, \boldsymbol{\eta}, \tau) \equiv \frac{1}{2 T} \int_{-T}^{T}\left[\rho v_{\lambda}^{\prime} v_{j}^{\prime}-\overline{\rho v_{\lambda}^{\prime} v_{j}^{\prime}}\right]\left(\boldsymbol{y}, \tau_{0}\right)\left[\rho v_{\kappa}^{\prime} v_{l}^{\prime}-\overline{\rho v_{\kappa}^{\prime} v_{l}^{\prime}}\right]\left(\boldsymbol{y}+\boldsymbol{\eta}, \tau_{0}+\tau\right) d \tau_{0}
$$

by the relatively simple linear transformation

$$
R_{\kappa j \lambda l}=\varepsilon_{\kappa j, \sigma m} R_{\sigma m \gamma n} \varepsilon_{\lambda l, \gamma n}
$$

where

$$
\varepsilon_{\kappa j, \sigma m} \equiv \delta_{\kappa \sigma} \delta_{j m}-\frac{\gamma-1}{2} \delta_{\kappa j} \delta_{\sigma m}
$$

when viscous effects are neglected. This result shows that there is an exact relation between the quantities that are actually measured in Aeroacoustics experiments: namely the far field pressure autocovariance and the generalized Reynolds stress autocovariance tensor. It is very general and applies to any localized turbulent flow even in the presence of fixed solid surfaces. The hope is that most of the non-local "propagation effects" 
which would be very difficult to distinguish from the turbulent fluctuations-and, therefore, very difficult to model— have been removed from these stresses.

\section{APPLICATIONS OF THE MEAN FLOW ANALOGY}

Karbasov et al (2008) used this form of the analogy to predict the sound from a cold Mach 0.75 jet and obtained reasonably good agreement with experimental data from the JEAN project. But the Green's function and, therefore, the propagator is much more expensive to compute (in fact prohibitively so for many purposes) than the corresponding Lilley equation propagator. There is, however, a way to obtain the best of both worlds, by using a perturbation approach that takes advantage of the small spread rate, say $\varepsilon$, of the jet or shear layer (Goldstein \& Leib, 2005, 2008). The base flow velocities will then expand like

$$
\begin{gathered}
\tilde{v}_{1}=U\left(Y, \boldsymbol{y}_{T}\right)+\varepsilon U^{(1)}\left(Y, \boldsymbol{y}_{T}\right)+\ldots \\
\tilde{\boldsymbol{v}}_{T}=\boldsymbol{\varepsilon} \boldsymbol{V}\left(Y, \boldsymbol{y}_{T}\right)+\varepsilon^{2} \boldsymbol{V}^{(1)}\left(Y, \boldsymbol{y}_{T}\right)+\ldots
\end{gathered}
$$

with similar expansions for the other dependent variables.

$$
Y \equiv \varepsilon y_{1}
$$

denotes a slow streamwise source variable that varies on the streamwise length scale of the mean flow (see figure1), and

$$
\begin{aligned}
& \boldsymbol{y}_{T}=\left\{y_{2}, y_{3}\right\} \\
& \tilde{\boldsymbol{v}}_{T}=\left\{\tilde{v}_{2}, \tilde{v}_{3}\right\}
\end{aligned}
$$

denote cross flow variables. I am assuming, of course, that all lengths have been normalized by some characteristic cross flow dimension of the jet and all velocities by some appropriate characteristic streamwise velocity with similar obvious normalization for the density, pressure and temperature. The lowest order terms in this expansion correspond to the unidirectional transversely sheared flow with the slow streamwise variable entering only parametrically.

The $4^{\text {th }}$ component of the adjoint Green's function is expected to possess a corresponding expansion of the form

$$
g_{v 4}^{a}=g_{v 4}^{a, 0}(\boldsymbol{y}, Y, \tau \mid \boldsymbol{x}, t)+\varepsilon g_{v 4}^{a, 1}(\boldsymbol{y}, Y, \tau \mid \boldsymbol{x}, t)+\ldots
$$


where the lowest order term satisfies the adjoint Rayleigh's equation in the vicinity of the source point. But this solution is non-uniformly valid because it still has a critical level singularity and becomes unbounded far downstream in the flow. However, the method of multiple scales, the WKBJ method and the method of matched asymptotic expansions can be combined to obtain a uniformly valid "composite" solution that remains finite everywhere in the flow and is still not much more expensive to compute than the original parallel flow solution. The resulting "propagator" consists of two components. The first of which corresponds to the usual non-causal Lilley equation solution appropriately corrected to eliminate the critical layer singularity. The second (causal component) is associated with linear instability waves, but differs from conventional instability models in that the instabilities, which are now continuously 'generated' along the length of the jet, do not constitute separate sound sources.

Previous attempts to incorporate instability waves into jet noise predictions have argued that there are two distinct sound sources: one associated with the smaller scale random motions and the other associated with the large scale coherent structures-with the latter source usually being represented by the linear instability waves. But recent studies show that the large scale motions are not well represented by these instabilities. The present approach avoids this conundrum because the instabilities only appear in the propagator with their sole function being to produce the appropriate cause-effect relation between the sound and its turbulent source. They do not correspond to any physical structures that actually appear in the flow.

Figure 2 (which is a compilation of data presented in Bridges et al) shows that the OASPL directivity patterns are smooth functions of angle at subsonic acoustic Mach numbers but exhibit a relatively abrupt increase in slope at about $60^{\circ}$ from the downstream axis at supersonic Mach numbers—-suggesting that some new effect may be coming into play at the smaller angles.

The non-causal component of the Green's function is expected to produce a relatively smooth directivity pattern, but results presented in Goldstein and Leib (2005) show that the causal component of the far field Green's function is highly damped at subsonic speeds and also at supersonic speeds when the observation angle (measured from the downstream axis) is sufficiently large. However the damping rapidly drops to zero when the observation angle become less than $60^{\circ}$ (see figures 4 through 6 of Goldstein and Leib, 2005) allowing the causal component of the Green's function to become much larger than the non-causal component. But source non-compactness effects can also produce an abrupt increase in shallow angle directivity. And Goldstein and Leib (2008) tentatively concluded that the causal component only becomes significant when the source point is close to the nozzle where reflection and diffraction effects are important and can, therefore, be neglected since the latter effects are already assumed to be negligible in the weakly non-parallel flow approach. They then used the remaining non-causal component (appropriately corrected for the critical layer singularity) to predict the sound from cold high speed jets over a range of acoustic 

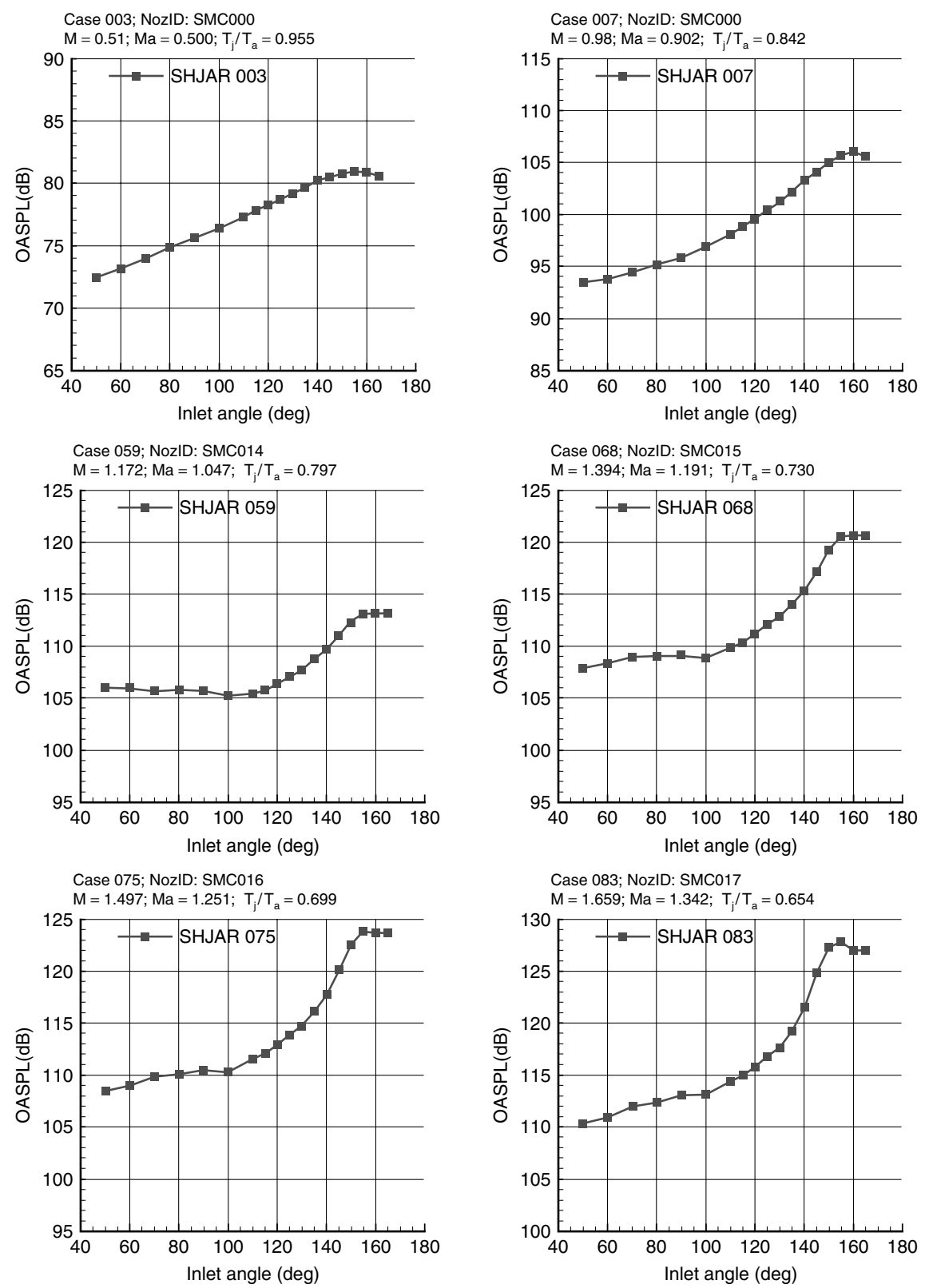

Figure 2: OASPL directivity patterns measured by Bridges on the Glenn SHJAR rig (Bridges et al, 2009). 
Mach numbers from 0.5 to 1.4 and were able to obtain reasonable agreement with measurements taken on the Glenn SHAR rig (Khavaran, Bridges \& Freund, 2002). These issues obviously need additional consideration

\section{CONCLUSIONS}

Lilley developed a modification of Lighthill's equation that explicitly separates the so called entropy source into its isentropic and non-isentropic components. The present paper shows how this result can be obtained as a special case of a generalized acoustic analogy that can be used to eliminate some of the difficulties that arise when more conventional parallel flow analogies are applied to high speed jets.

\section{ACKNOWLEDGEMENT}

This work was supported by NASA's supersonic fixed wing project.

\section{REFERENCES}

[1] Aldama, A.A. (1990) Filtering Techniques for Turbulent Flow Simulation (chapter 3) Springer-Verlag lecture Notes in Engineering.

[2] Bers, A. 1975 Linear waves and instabilities. In Plasma Physics (ed. C.DeWitt \& J. Perraud ), pp. 113-2166 Gordon \& Beach.

[3] Betchov, R. and Criminale, W.O. (1967) Stability of parallel flows, Academic Press.

[4] Bodony, L.J. and Lele, S.K. (2002) Spatial scale decomposition of shear layer turbulence and the sound sources associated with the missing scales in a largeeddy simulation, AIAA Paper No. 2002-2454, $8^{\text {th }}$ AIAA/CEAS Aeroacoustics Conference, Breckenridge, $\mathrm{CO}$.

[5] Bodony, D.J. and Lele, S.K. (2003) A Stochastic Subgrid Scale Model for Noise Predictions of Subsonic Jets, AIAA Paper No. 2003-3252, $9^{\text {th }}$ AIAA/CEAS Aeroacoustics Conference, Hilton Head, South Carolina.

[6] Bodony, D.J. and Lele, S.K., (2005) On using large-eddy simulation for the prediction of noise from cold and heated turbulent jets, Phys. Fluids 17, 085103 (20 pages).

[7] Bridges, J. E., Khavaran, A. and Hunter, C. A., (2009) Jet Noise Prediction, Chapter 8 in Assessment of NASA's Noise Prediction Capability, NASA/TP20XX-215653, Dahl, M. D. (ed.), to be published

[8] Briggs, R. J. 1964 Electron Stream Interaction with Plasmas. Massachusetts Institute of Technology Press.

[9] Crighton, D.G. and Leppington, F. G., 1974, Radiation properties of the semiinfinite vortex sheet: the initial value problem, Journal of Fluid Mechanics, vol. 64, part 2 , pp. 393-414.

[10] Crighton, D.G., (1985) The Kutta condition in unsteady flow, Annual Review of Fluid Mechanics, vol. 17 pp. 411-446. 
[11] Dowling, A.P., Ffowcs Williams, J.E. and Goldstein, M.E., (1978) Sound propagation in a moving stream, Phil. Trans. Roy. Soc. Lond., A, vol. 288, no. 1353, pp. 321-349.

[12] Freund, J.B. (2001) Noise sources in a low-Reynolds-number turbulent jet at Mach 0.9, J. Fluid Mech., vol. 438, pp. 277-305.

[13] Goldstein, M.E., (1976), Aeroacoustics, McGraw-Hill.

[14] Goldstein, M.E. (1999), Some recent developments in jet noise modeling, Program and Abstracts of the $6^{\text {th }}$ International Congress on Sound and Vibration, Copenhagen, Denmark, p. 21.

[15] Goldstein, M.E. (2000), Some recent developments in jet noise modeling, Program of the $38^{\text {th }}$ AIAA Aerospace Sciences Meeting and Exhibit, Reno, Nevada.

[16] Goldstein, M.E. (2001), “An exact form of Lilley's equation with a velocity quadrupole/temperature dipole source term," Journal of Fluid Mechanics, vol. 443, pp. 231-236.

[17] Goldstein, M.E. (2002), A unified approach to some recent developments in jet noise theory, International Journal of Aeroacoustics, vol. 1, no. 1, pp. 1-16. Reprinted in Jet Aeroacoustics, 2008, pp 1-17. Ganesh Raman ed., Multi-Science Publishing Company Ltd.

[18] Goldstein, M.E. (2003), "A Generalized Acoustic Analogy," Journal of Fluid Mechanics, vol. 488, pp. 315-333.

[19] Goldstein, M.E. (2005), "On identifying the true sources of aerodynamic sound," Journal of Fluid Mechanics, vol. 526, pp. 3337-347.

[20] Goldstein, M.E. (2006), "Hybrid Reynolds-averaged Navier-Stokes/Large eddy simulation approach for predicting jet noise" AIAA Journal vol. 44, no. 12 , pp. $3136-3142$.

[21] Goldstein, M.E. (2008), A theoretical basis for identifying the sound sources in a turbulent flow, to be published, International Journal of Aeroacoustics,

[22] Goldstein, M.E. and Leib, S.J. (2005) The Role of Instability Waves in Predicting Jet Noise, J. Fluid Mech. vol. 525, pp. 37-72.

[23] Goldstein, M.E. and Leib, S.J. (2008), The Aeroacoustics of slowly diverging supersonic jets , J. Fluid Mech. vol. 600, pp. 291-337.

[24] Karabasov, S. A., Afsar, M. Z., Hanes, T. P., Dowling, A.P., McMullan, W. A., Pokora, C. D., Page, G. J.,\& McGuirk, J. J., 2008, Using Large Eddy Simulation within an Acoustic Analogy Approach for Jet Noise Modeling, 14 ${ }^{\text {th }}$ AIAA/CEAS Aeroacoustics Conference, Vancouver, British Columbia.

[25] Khavaran, A., Bridges, J. and Freund, J. (2002), A Parametric study of fine scale Turbulence mixing Noise, NASA TM 2002-211696.

[26] Khavaran, A and Kenzakowski, D. C., 2007, Progress Toward Improving Jet Noise Predictions in Hot Jets, AIAA paper \# 2007-0012.

[27] Lighthill, M.J. (1952) On sound generated aerodynamically: I. General theory, Proc. R. Soc. Lond., A211, pp. 564-587. 
[28] Lighthill, M.J. (1954) On sound generated aerodynamically: II. Turbulence as a source of sound, Proc. R. Soc. Lond. A222, pp. 1-32.

[29] Lighthill, M.J., (1963), 'Jet noise', The 1963 Wright Brothers Lecture, AIAA Journal, vol. 1, pp. 1507-1517.

[30] Lilley, G.M. (1974) On the noise from jets, Noise Mechanism, AGARD-CP-131, pp. 13.1-13.12.

[31] Lilley, G.M. (1996) The radiated noise from isotropic turbulence with applications to the theory of jet noise, J. Sound and Vib., vol. 190, no. 3, pp. 463-476.

[32] Lilley, G.M. (2003) The source of aerodynamic noise, International Journal of Aeroacoustics, vol. 2, no.3 \& 4 , pp. 241-254. Reprinted in Jet Aeroacoustics, 2008, pp. 19-32, Ganesh Raman ed., Multi-Science Publishing Company Ltd.

[33] Morse, P. M. \& Fesbach, H.,1953, Methods of Theoretical Physics. McGraw-Hill, p.870

[34] Pridmore-Brown, D.C. (1958) Sound propagation in a fluid flowing through an attenuating duct J. Fluid Mech., vol. 4, pp. 393-406.

[35] Tam, C.K.W. \& Auriault, L. 1998 Mean Flow Refraction Effects on Sound Radiated from Localized Sources in a Jet. J. Fluid Mech. 370 pp. 149-174.

[36] Yih, C-S, 1977, Fluid Mechanics, West River Press, Ann Arbor, Michigan. 